\title{
Ferroelectric engineering: Enhanced thermoelectric performance by local structural heterogeneity
}

\author{
Xiangyu Meng ${ }^{1,2}$, Shuo Chen ${ }^{1}$, Haoyang Peng ${ }^{1,2}$, Hui Bai ${ }^{1,2}$, Shujun Zhang ${ }^{3}$, Xianli Su ${ }^{1}$, Gangjian Tan ${ }^{1}$, \\ Gustaaf Van Tendeloo ${ }^{2,4}$, Zhigang Sun ${ }^{1}$, Qingjie Zhang ${ }^{1}$, Xinfeng Tang ${ }^{1}$ and Jinsong $\mathrm{Wu}^{1,2^{*}}$
}

\begin{abstract}
Although traditional ferroelectric materials are usually dielectric and nonconductive, $\mathrm{GeTe}$ is a typical ferroelectric semiconductor, possessing both ferroelectric and semiconducting properties. GeTe is also a widely studied thermoelectric material, whose performance has been optimized by doping with various elements. However, the impact of the ferroelectric domains on the thermoelectric properties remains unclear due to the difficulty to directly observe the ferroelectric domains and their evolutions under actual working conditions where the material is exposed to high temperatures and electric currents. Herein, based on in-situ investigations of the ferroelectric domains and domain walls in both pure and Sb-doped GeTe crystals, we have been able to analyze the dynamic evolution of the ferroelectric domains and domain walls, exposed to an electric field and temperature. Local structural heterogeneities and nano-sized ferroelectric domains are generated due to the interplay of the $\mathrm{Sb}^{3+}$ dopant and the Ge-vacancies, leading to the increased number of charged domain walls and a much improved thermoelectric performance. This work reveals the fundamental mechanism of ferroelectric thermoelectrics and provides insights into the decoupling of previously interdependent properties such as thermo-power and electrical conductivity.
\end{abstract}

Keywords: charged domain walls, bound charge, local structural heterogeneity, high-performance thermoelectric

\section{INTRODUCTION}

The traditional view of a ferroelectric is that the spontaneous polarization can be switched by an applied external electric field [1-6]. The switching of polarity is normally realized by the electrostatic force which can be directly applied to an insulating ferroelectric material. In a conductor or a semiconductor with a small band gap, the force is screened out owing to the freely moving electrons/holes. Therefore, ferroelectric metals have not been confirmed until recently, although their existence was predicted in 1968 [7-10]. Although ferroelectric semiconductors have been reported for a long time, the number of known materials with dual properties remains few [11-19]. Ferroelectric semiconductors not only exhibit spontaneous polarization but also show a unique electrical conductivity. Therefore, they have attracted extensive attention in the development of functional apparatus, such as sensors or energy harvesting and conversion devices.

Semiconductors when used as thermoelectric materials can convert waste heat into electricity or use electricity for cooling without air pollution. The energy conversion efficiency is defined by the figure of merit $Z T=\left(S^{2} \sigma / \kappa\right) T$, where $S, \sigma$, $T$, and $\kappa$ are the Seebeck coefficient, electrical conductivity, absolute temperature, and thermal conductivity, respectively [20-25]. It is challenging to search for new materials with an improved $Z T$, since the Seebeck coefficient $(S)$, the electric $(\sigma)$ and thermal transport $(\kappa)$ parameters are interrelated, which can be enhanced only at the expense of each other [26-28]. Ferroelectric semiconductors bring an additional ordering, namely the electric polarization, into the structure design. It is thus informative to explore the impact of the ferroelectricity to the thermoelectrics.

Germanium telluride $(\mathrm{GeTe})$ is a semiconductor with a narrow band gap, and a high-performance thermoelectric material at medium-temperature. It exhibits a ferroelectric phase transition around $700 \mathrm{~K}$ (Curie temperature) from a face-centered cubic to a rhombohedral structure, with a lattice elongation where Ge is displaced off the center along the [111] direction [29]. Below the Curie temperature, GeTe exhibits simultaneously thermoelectricity and ferroelectricity with a typical herringbone domain structure [30]. Recently, theoretical simulations predicted a significant increase of the Seebeck coefficient at the $39^{\circ}$ and $141^{\circ}$ charged domain walls (CDWs) in GeTe, without a significant deterioration of the electrical conductivity [31].

Thermoelectrics are exposed to a broad temperature range and high electric current (or electric field) when they are used as either a thermo-power generator, or a solid state cooler. However, the dynamic evolution of the ferroelectric domains in a ferroelectric semiconductor under an applied electric field (i.e., a current) remains unexplored. With the recent development of in-situ experimental techniques, the structural evolution can be

\footnotetext{
${ }^{1}$ State Key Laboratory of Advanced Technology for Materials Synthesis and Processing, Wuhan University of Technology, Wuhan 430070, China

${ }^{2}$ Nanostructure Research Center, Wuhan University of Technology, Wuhan 430070, China

${ }^{3}$ Institute for Superconducting and Electronic Materials, Australian Institute for Innovative Materials, University of Wollongong, Wollongong, NSW 2500, Australia

${ }^{4}$ EMAT (Electron Microscopy for Materials Science), University of Antwerp, Belgium

*Corresponding author (email: wujs@whut.edu.cn)
} 
observed in conditions close to the real applications. Herein, we study the local structure of the ferroelectric domains and domain-walls in thermoelectric GeTe and Sb-doped GeTe at an atomic scale by scanning transmission electron microscopy (STEM). The effects of the ferroelectric domains and DWs on the experimentally measured properties, e.g., electrical and thermal conductivities, and thermal power were then analyzed (at temperatures below the Curie temperature $\sim 700 \mathrm{~K}$ ). This work provides a new paradigm to improve the thermoelectric properties of semiconductor materials by means of ferroelectric domain engineering through effective elemental doping.

\section{EXPERIMENTAL SECTION}

\section{Materials}

High-purity raw elements Ge (99.99\%), Te (99.99\%) and $\mathrm{Sb}$ (99.99\%) were weighed according to the nominal ratios of GeTe and $\mathrm{Sb}_{0.08} \mathrm{Ge}_{0.88} \mathrm{Te}$ and enclosed in fused silica tubes. The mixed raw materials were heated from room temperature to $1273 \mathrm{~K}$ at a rate of $2 \mathrm{~K} \mathrm{~min}^{-1}$. After being kept at this temperature for $24 \mathrm{~h}$, they were quickly quenched in saturated brine, then heated from room temperature to $773 \mathrm{~K}$ at a rate of $2 \mathrm{~K} \mathrm{~min}^{-1}$, and annealed at this temperature for three days. The obtained ingots were manually ground into fine powders. We used a spark plasma sintering (SPS) device to vacuum sinter the samples at $773 \mathrm{~K}$, $50 \mathrm{MPa}$ for $5 \mathrm{~min}$ to obtain a relative density up to $95 \%$ of the bulk samples.

\section{Instrumentation}

The TEM samples were prepared by focused ion beam (FIB) (Helios Nanolab G3 UC, FEI) for structural characterization and in-situ TEM observation. The in-situ TEM was carried out using a $200-\mathrm{kV}$ transmission electron microscope (Talos F200s, FEI), with a PicoFemto double-tilt TEM-STM electric holder and a Dens Solution in-situ biasing and heating holder. The atomic resolution high-angle annular dark field (HAADF)-STEM images were obtained using a $300 \mathrm{kV} \mathrm{C}$-corrected transmission electron microscope (Titan Themis G2 60-300, FEI). A series of images were continuously taken and then drift-corrected to reduce the sample drift during the acquisition process. The electrical conductivity and the Seebeck coefficient were measured using a ZEM-3 apparatus (Ulvac Riko, Inc.) under a helium atmosphere from 300 to $823 \mathrm{~K}$. The thermal conductivity was determined from the equation $\kappa=\lambda \rho C_{\mathrm{p}}$, where $\lambda$ is the thermal diffusivity and measured in an argon atmosphere by the laser flash method (LFA 457; Netzsch), $C_{\mathrm{p}}$ is the specific heat capacity calculated by the Dulong-Petit law, and $\rho$ is the sample density determined by Archimedes' method.

\section{DFT calculations}

All density function theory (DFT) calculations were carried out within the periodic plane wave framework as implemented in the Vienna $a b$ initio simulation package (VASP). The electronion interaction was represented by the projector augmented wave (PAW), the kinetic energy cutoff of plane wave was set as $520 \mathrm{eV}$. The geometry optimization was based on the exchangecorrelation functional GGA-PBE (generalized-gradient approximation-Perdew-Burke-Ernzerhof). The geometry convergence criterion was set as $0.02 \mathrm{eV} \AA^{-1}$ for the maximal component of force. The k-point mesh utilized was up to $3 \times 3 \times 5$ in the gamma centered Monkhorst-Pack grid.

\section{RESULTS AND DISCUSSION}

\section{Ferroelectric domains and DWs related to the Ge-vacancy gaps in GeTe}

The ferroelectric domains and domain-walls in pure GeTe were carefully studied by STEM images collected by an HAADF detector. Owing to the symmetry break, spontaneous ferroelectric domains with eight possible polarization vectors (along the [111] directions) are formed to release the stress during the phase transition. Considering the angle between polarization vectors in neighboring domains, three types of domain-walls, namely $71^{\circ} / 109^{\circ}, 180^{\circ}$ and $141^{\circ} / 39^{\circ}$, were found as shown in Fig. S1 and Fig. 1. The domain-wall plane can be one of the $\{111\},\{110\}$ and $\{100\}$ lattice planes (referring to the pseudocubic unit cell). Based on the orientation of the polarization vectors in adjacent domains, three configurations, namely headto-head, tail-to-tail and head-to-tail, are possible. The typical herringbone domain structure with $71^{\circ} / 109^{\circ}$ DWs in the $\{111\}$ and $\{110\}$ planes with both $\mathrm{H}-\mathrm{H}$ and $\mathrm{T}-\mathrm{T}$ configurations (Fig. S1a) is the most observed DW in pure GeTe, in agreement with the previous results $[29,30]$. The spontaneous polarization in the ferroelectric domains can be directly imaged by the differential phase contrast (DPC) method, as shown in Fig. S1b, in which the orientation of the domains can be seen and the $109^{\circ}$ domain-wall can be identified. The polarization vector in a domain can also be directly measured as the deviation of the $\mathrm{Ge}$ from its position in the Te-polyhedron. This displacement can be measured from the atomic resolution STEM images, as shown in Fig. 1c, which is an HAADF-STEM image of GeTe taken along the [0-11] zone axis. In Fig. 1a, c, the yellow arrows indicate the local polarization vectors from the geometric centers of the Te parallelograms to the Ge atoms; they express both the magnitude and direction of the polar vector. Although they are a minority in the pure GeTe, $180^{\circ} \mathrm{DW}, 141^{\circ} \mathrm{DW}$ with both $\mathrm{H}-\mathrm{H}$ and T-T configurations are found as well (Fig. S1c). It reflects the complex approach of relaxing the stress in a real sample. Naturally, between two T-T $141^{\circ}$ DWs there will always be an $\mathrm{H}-\mathrm{H} 141^{\circ} \mathrm{DW}$, as shown in Fig. 1a, which has been identified as pairs of the van der Waals gap and charged nano-domain [32]. Although some of the $141^{\circ}$ DWs are not straight (Fig. S2c), the DW plane is actually still along the $\{111\}$ plane and such a curved wall is a sliding through the atomic layer as shown in Fig. S2. And there is a large strain around the $\mathrm{H}-\mathrm{H} 141^{\circ} \mathrm{CDWs}$ as shown in Fig. S3.

The DW can be neutral or positively/negatively charged, depending on the orientation relationship of the adjacent domains and their polar vectors. The magnitude of the electric field experimentally measured by DPC (Fig. 1b, d) shows that the maximum value is along the $\mathrm{T}-\mathrm{T} 141^{\circ} \mathrm{DW}$, and the minimum value is along the $\mathrm{H}-\mathrm{H} 141^{\circ} \mathrm{DW}$. The $\mathrm{T}-\mathrm{T} 141^{\circ} \mathrm{DW}$ accumulates positive charges while the $\mathrm{H}-\mathrm{H} 141^{\circ} \mathrm{DW}$ is negatively charged. This agrees with the theoretical analysis, which suggests that $\mathrm{H}-\mathrm{H}$ and T-T DWs are charged [14,33-37]. As $\mathrm{GeTe}$ is a p-type semiconductor whose carriers are holes, the negative $\mathrm{Ge}$ vacancies tend to accumulate along the positively charged $\mathrm{T}-\mathrm{T} 141^{\circ} \mathrm{DW}$, forming a so-called van der Waals vacancy plane (Fig. 1c), with an enhanced electric field magnitude, shown in Fig. 1d. An illustration of the accumulated extra charges at T-T and $\mathrm{H}-\mathrm{H} 141^{\circ} \mathrm{DWs}$ is shown in Fig. S4. The DWs with a head-to-tail configuration are electrically neutral, such as 

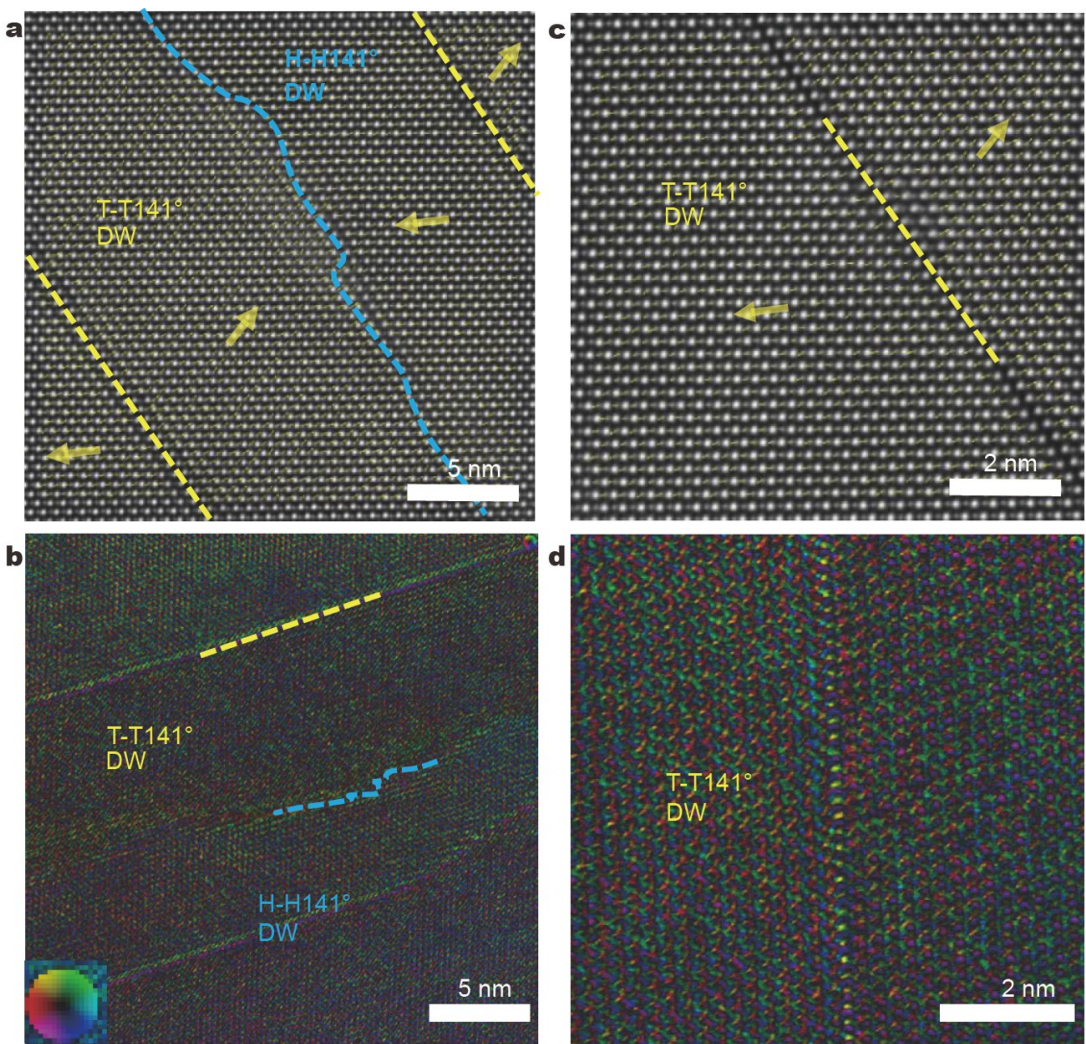

Figure 1 Ferroelectric domains and DWs in pure GeTe. (a) An HAADF-STEM image of H-H $141^{\circ}$ DWs between a T-T $141^{\circ}$ DW. (b) A DPC image of the charged DWs. (c, d) Enlarged HAADF and DPC images of a T-T $141^{\circ}$ DW, clearly showing the electric field distribution.

the $109^{\circ} \mathrm{DW}$ and the $180^{\circ} \mathrm{DW}$ (Fig. S1c).

\section{Sb-doping and Ge-vacancy forming the local structural heterogeneity in GeTe}

The doping of $\mathrm{Sb}$ reduces the electron hole concentration and thus the carrier concentration. Doping by $\mathrm{Sb}$ also alters the spatial distribution of the Ge-vacancies with the formation a local structural heterogeneity, although the Ge-vacancy level remains high as reported [38]. The local structural heterogeneity introduced by $\mathrm{Sb}^{3+}$ doping was experimentally identified in this research as the source of a reduced domain size and an increased number of CDWs. The width of the domains in pure GeTe is about $\sim 100 \mathrm{~nm}$, while in the Sb-doped GeTe it is reduced to about $\sim 5 \mathrm{~nm}$ (Fig. 2a). The Sb-doping greatly reduces the domain size and creates a large number of $141^{\circ} \mathrm{DWs}$. In contrast, large domains are found in the pure GeTe. The distance from the $\mathrm{H}-\mathrm{H} 141^{\circ} \mathrm{DW}$ to the $\mathrm{T}-\mathrm{T} 141^{\circ} \mathrm{DW}$ is now about $\sim 5 \mathrm{~nm}$ (Fig. 2a), implying that the local electric fields due to the charged DWs is greatly enhanced.

DFT simulations show that the Sb dopant prefers to occupy the Ge-vacancies; these Ge-vacancies already exist in pure GeTe, as shown in Fig. S5. Then it tends to gather more cationvacancies in the doped area, forming a Sb-dopant and cationvacancy intercalated structure. As shown in Fig. S6, the formation energy for a Sb dopant to occupy a Ge-vacancy or to replace a Ge close to a vacancy is negative, which is an energy favorable path. All other ways for Sb to enter the GeTe lattice result in unfavorable paths with a positive formation energy. Moreover, the shorter the distance between $\mathrm{Sb}$ and the vacancy, the smaller
$\Delta E$ (which is the difference between the initial energy and the resulting energy); this is shown in Fig. $2 \mathrm{~b}$. It implies that the vacancy will be attracted by the doped Sb and there is a tendency to form a cation-vacancy concentrated area around the doped $\mathrm{Sb}$.

Such a dopant and vacancy intercalated structure generates a large variation in the strain distribution (i.e., fast switching from compressive to tensile strain), and it was confirmed by STEM observations. It is also the underlying reason for the creation of a local structural heterogeneity [39]. In the polarization vector mapping of Sb-doped GeTe (Fig. 2 $c_{1}$ ), there is an area (outlined by the black square) having a high concentration of vacancies and dopants (reflected by the low column intensity shown in Fig. $2 c_{2}$ ), large variations of polarization intensity and angle (Fig. $2 c_{3}, c_{4}$ ), and a large varying strain distribution (Fig. S7). From the enlarged STEM image of the square area with the marked polarization (Fig. $2 c_{5}$ ), some strange singular points (similar to anti-vortex) were observed. Around the point, the two T-T polarization vectors converge toward the center, while the other $\mathrm{H}-\mathrm{H}$ polarization vectors diverge away from the center (as shown in Fig. $2 c_{5}$ ). Based on a quantitative analysis of the Gesite intensity as shown in Fig. $2 c_{2}$ (which reflects the presence of a Sb-dopant and thus the generation of a Ge-vacancy), the singular points have a relatively low atomic column intensity, implying a large number of Ge-vacancies due to Sb doping, in agreement with the DFT results. Similar singular points (termed as local structural heterogeneities) were found over the entire area with a large variation of the strain distribution, as shown in Fig. 2d. The walls of the four nano-domains of the local struc- 
a

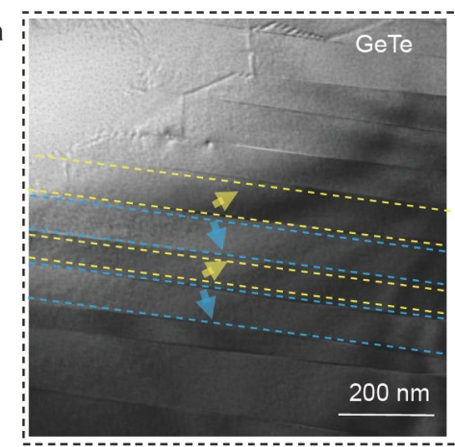

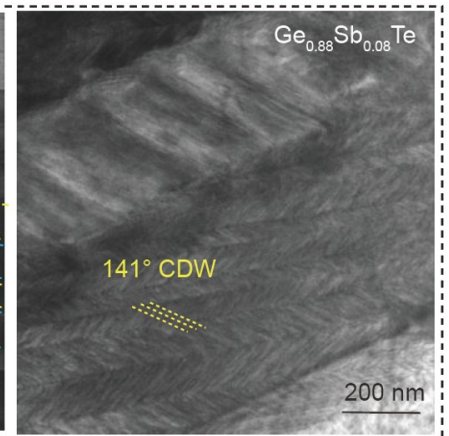

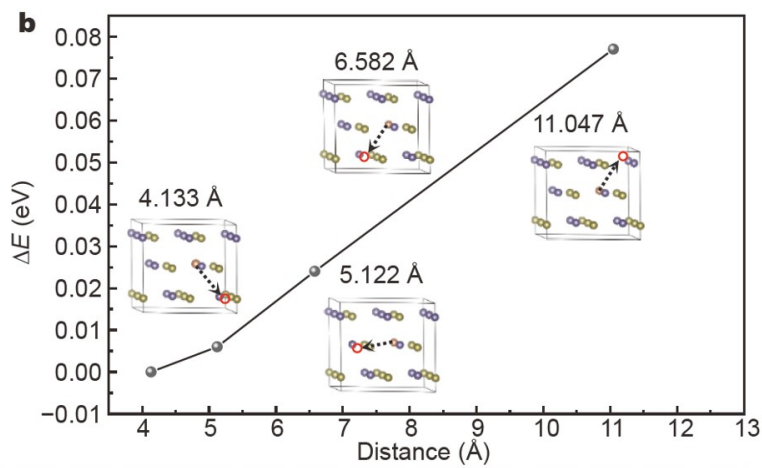

c
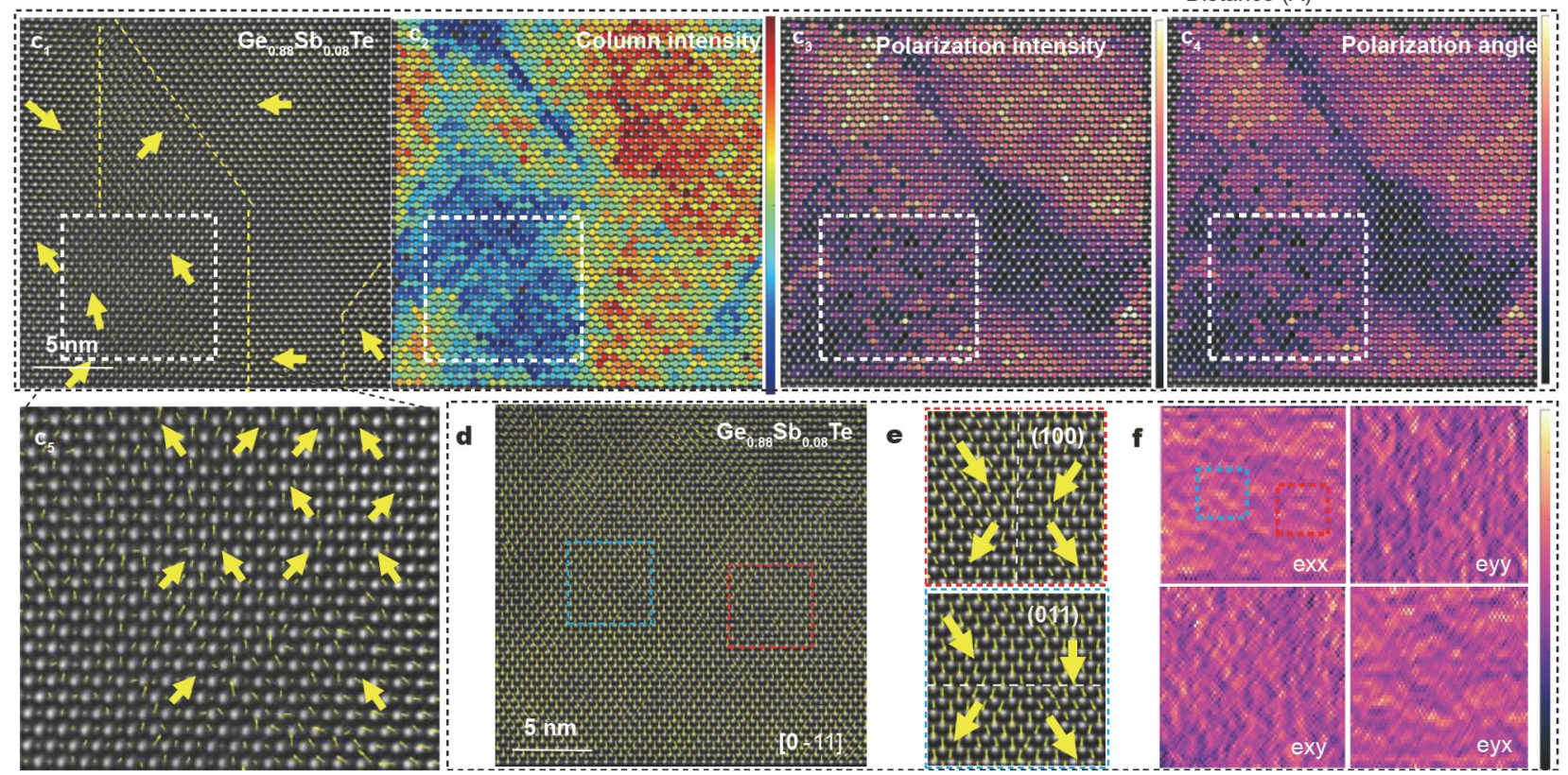

Figure 2 Local structural heterogeneities in Sb-doped GeTe. (a) Bright field TEM images of GeTe and $\mathrm{Ge}_{0.88} \mathrm{Sb}_{0.08} \mathrm{Te}$, respectively, showing a higher density of DWs due to the Sb-doping. (b) Difference between the initial and resulting energy, $\Delta E$ (eV), calculated by DFT versus the distance between the Ge vacancy and the Sb dopant. (c) HAADF image of the doped GeTe showing a specific area outlined by the square, with a high concentration of vacancies and dopants $\left(c_{2}\right)$, large variations of polarization intensity $\left(c_{3}\right)$ and angle $\left(c_{4}\right)$ and the configuration of the $\{100\}$ nano-domains forming the local structural heterogeneity $\left(c_{5}\right)$. (d) The local structural heterogeneity, (e) strange singular points marked by the square area in (d) and (f) its strain maps observed in another area.

tural heterogeneity are along the $\{100\}$ lattice plane (Fig. 2e), accompanied by a large variation of strain (Fig. 2f). The $\{100\}$ DWs are more efficient for strain release, although they have a much higher formation energy compared with the $\{110\}$ and $\{111\}$ planes. Similar results were obtained from other areas collected in different samples as shown in Fig. S8, demonstrating a high reproducibility. With the formation of a large number of local structural heterogeneities, various and more complicated DW configurations are observed, prompting the formation of high density CDWs, as shown in Fig. S9. Therefore, the microstructural change of the domains and DWs due to $\mathrm{Sb}^{3+}$ doping is believed to originate from the local structural heterogeneities, which refers to the nano-sized (or unit cell scaled) polarization in local regions due to the atomic strain disturbance.

\section{Evolutions of the ferroelectric domains when exposed to an applied electric field and high temperatures}

The dynamic evolution of the ferroelectric domains in pure GeTe under an external applied electric field has been studied by in-situ TEM. With an increasing voltage (from 0 to $1.9 \mathrm{~V}$ ), there are changes in both image contrast (marked in Fig. 3a) and angle between the related diffraction spots over the two domains (listed in Supplementary information Table S1). Although the angle between symmetry related reflections can slightly alter due to the external electric field, the polarization reversal or domain switching along the external electric field direction does not occur because of its high conductivity. Also the DWs are relatively stable, implying that the extra resistance (i.e., the voltage drop due to the DWs) is relatively small and has a minimal impact on the re-orientation of the ferroelectric domains. However, the splitting angle of the spots has a tendency of deceasing with increasing voltage (Table S1), implying that the rhombohedral distortion is gradually decreasing, probably due to the temperature rising from Joule heating.

On the contrary, the ferroelectric domains and the DWs in the $\mathrm{Sb}$-doped GeTe show a clear evolution with electric field, especially in the regions with a large number of local structural heterogeneities. As shown in Fig. 3b, a vortex appears at an applied voltage of $0.1 \mathrm{~V}$, along with the appearance of small domains $(\sim 50 \mathrm{~nm})$ whose polarization is along the external electric field direction. This demonstrates that the resistance in such local structural heterogeneities is high and thus the voltage drop is large enough to trigger a local polarization reversal. With 


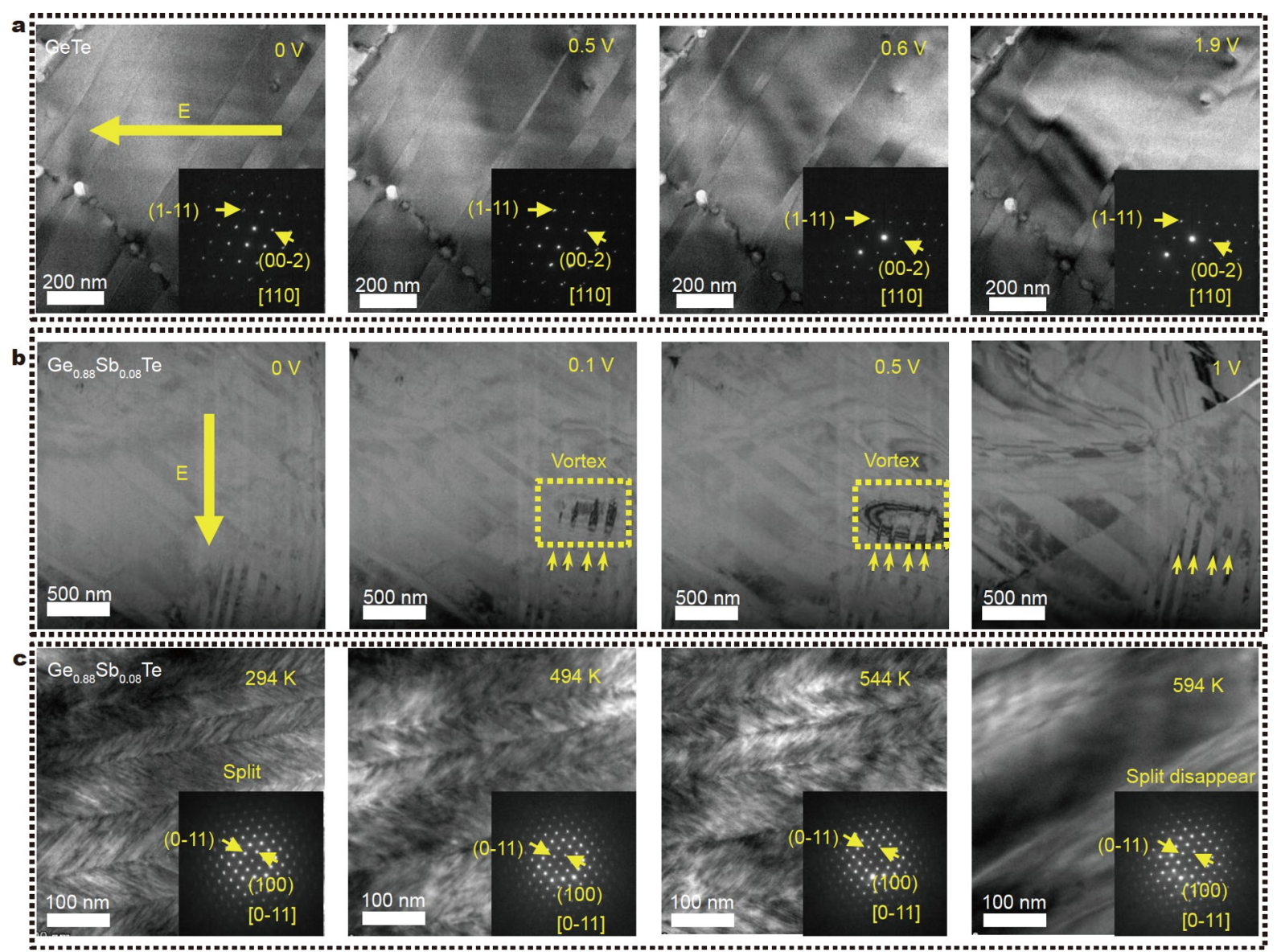

Figure 3 Evolutions of the ferroelectric domains and DWs under the applied voltage and temperature. (a) A series of TEM images and selected area electron diffraction (SAED) patterns of pure GeTe under an applied voltage from 0 to 1.9 V. (b) A series of TEM images of Sb-doped GeTe under an applied voltage from 0 to 1 V. (c) A series of TEM images and SAED patterns along the [001] direction of Sb-doped GeTe under elevated temperatures from 294 to 594 K; the ferroelectric domains disappear around the Curie temperature.

the applied voltage reaching $1 \mathrm{~V}$, larger domains $(\sim 120 \mathrm{~nm})$ are formed along the electric field direction and the vortex (as an intermedium transition state) has disappeared. Generally, the larger domains with low electric resistance remain stable, while the areas with small and immature domains are subject to polarization reversal.

With increasing temperature, the rhombohedral distortion in Sb-doped GeTe gradually decreases, and completely disappears above the Curie temperature. As shown in Fig. 3c, the splitting angle of diffractions gradually decreases with increasing temperature. The spot splitting disappears around $594 \mathrm{~K}$, the Sbdoped GeTe Curie temperature, which is lower than that of pure GeTe [40]. Above the Curie temperature, the Sb-doped GeTe transforms into a non-polar cubic phase and the ferroelectric domains (and DWs) disappear. Additional STEM and TEM images of the gradual disappearing are shown in Figs S10 and S11.

\section{Impact of the ferroelectric domains on the thermoelectric properties}

Ferroelectric engineering is an additional and effective approach to modulate the key parameters which are normally interrelated in thermoelectric semiconductors and improve their performance. As illustrated in Fig. 4a, b, a comparison of the ferroelectric domains and DWs in pure and doped GeTe, the local structural heterogeneity formed by Sb-doping and Ge-vacancy clusters in Sb-doped GeTe induces a localized point-like electrostatic field. Meanwhile, in the regions between the $\mathrm{H}-\mathrm{H} 141^{\circ}$ DW and the T-T $141^{\circ} \mathrm{DW}$, a few-nanometer scale electrostatic field is also formed. As large numbers of charged DWs are formed in the doped sample due to the local structural heterogeneities, such electrostatic field becomes much stronger than in the pure GeTe. Such a combined intrinsic electrostatic field greatly enhances the Seebeck coefficients as shown in Fig. 4c. Although the local structural heterogeneity has a localized high electric resistance, the high electric conductive paths remains in their surrounding areas with larger domains. This induces a moderate decrease of the overall electric conductivity, as shown in Fig. 4d. It represents a significant step to enhance the thermal power with a moderate decrease of electric conductivity, as proved by the measured thermal power, shown in Fig. 4f. Meanwhile, the DWs and boundaries of the local structural heterogeneities are effective in scattering the phonons and therefore have a great impact on reducing the thermal conductivity (Fig. 4e). All of the thermoelectric properties show different behaviors at temperatures above their respective Curie temperature, demonstrating the impact of the ferroelectric domains and DWs and local structural heterogeneities to the thermoelectric performance. The response to an applied electric field in the local structural heterogeneity regions is more sen- 


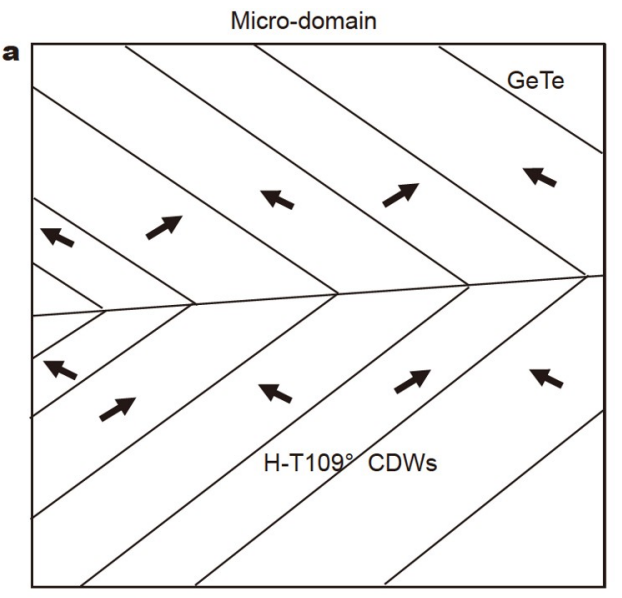

$500 \mathrm{~nm}$
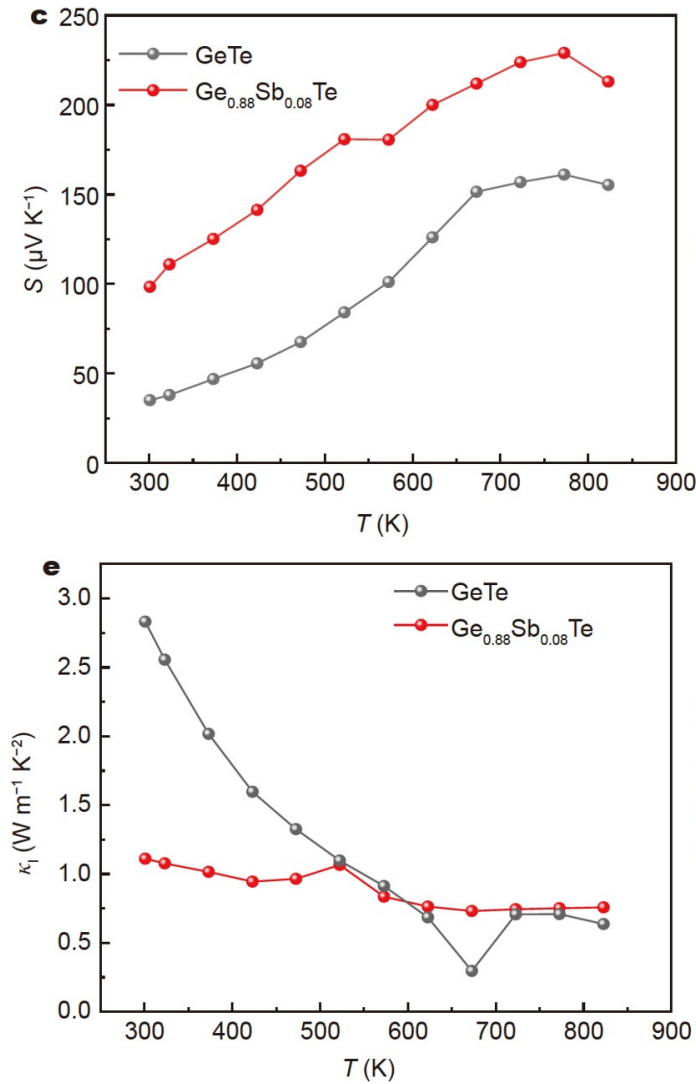

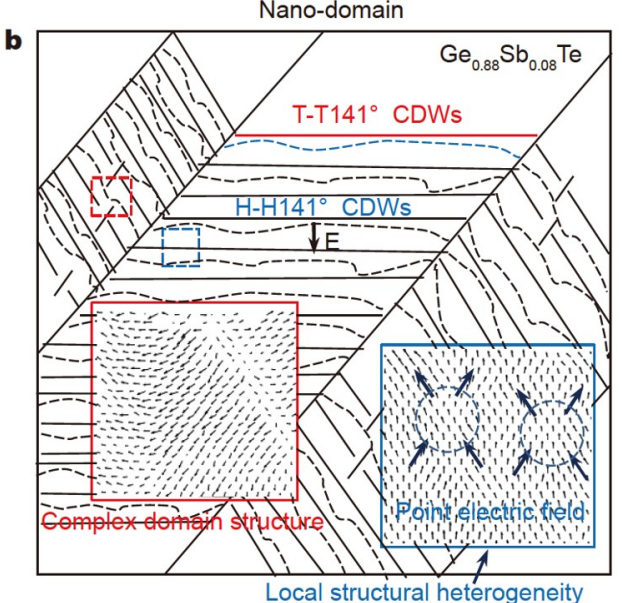

Local structural heterogeneity
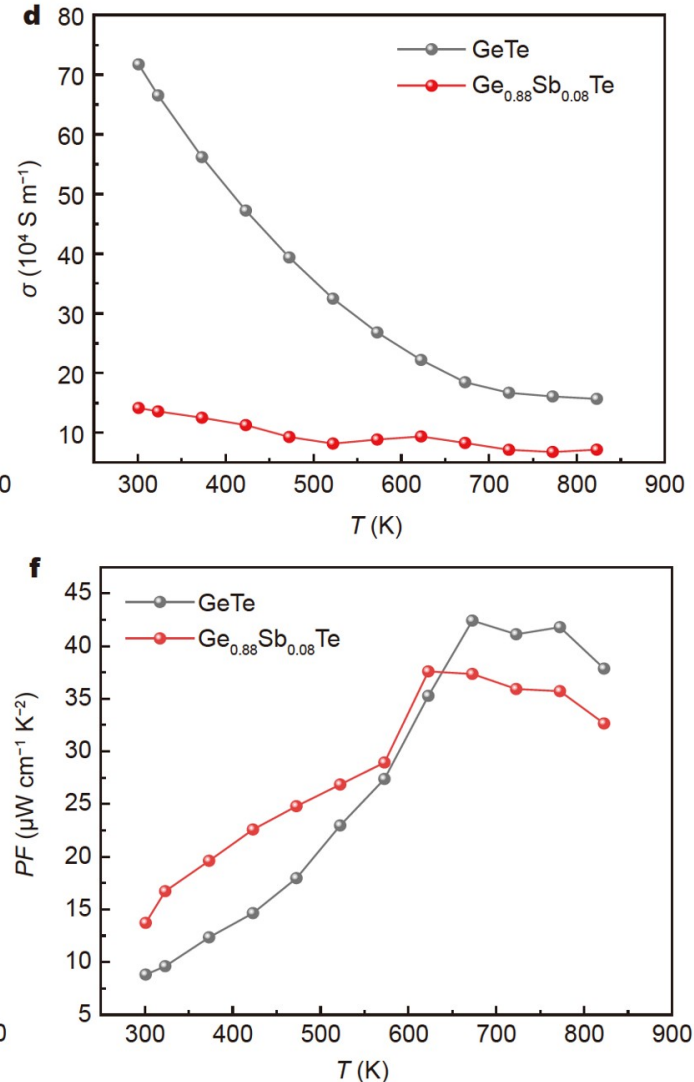

Figure 4 Impact of the ferroelectric domains and $\mathrm{DWs}$ on the thermoelectric properties, and comparison between pure $\mathrm{GeTe}_{\mathrm{T}}$ and $\mathrm{Ge}_{0.88} \mathrm{Sb}_{0.08} \mathrm{Te}$. $(\mathrm{a}, \mathrm{b})$ schematic diagram of the domain structures of GeTe and $\mathrm{Ge}_{0.88} \mathrm{Sb}_{0.08} \mathrm{Te}$. Comparison of (c) Seebeck coefficient $S$, (d) electric conductivity $\sigma$, (e) lattice thermal conductivity $\kappa_{1}$ and (f) power factor $\sigma S^{2}$ from 300 to $825 \mathrm{~K}$, of pure and doped GeTe, respectively.

sitive than in the already developed larger domains. This may lead to dynamically formed domains and DWs with a preferred orientation under real applications, which may explain the low thermal conductivity of Sb-doped GeTe (as shown in Fig. S12a). Due to the local structural heterogeneities, the $Z T$ of the doped sample is much higher than that of pure GeTe over the entire temperature range from 300 to $850 \mathrm{~K}$ as shown in Fig. S12b; especially in the medium temperature range when the ferroelectric domains and DWs are still present. For instance, at $470 \mathrm{~K}, Z T=0.69$ was achieved in Sb-doped GeTe compared with $Z T=0.16$ in the pure GeTe, which is an almost $~ 430 \%$ improvement. It is therefore clear that ferroelectric engineering may open a new pathway in the design of practical thermoelectric materials, appropriate for near room-temperature applications.

\section{CONCLUSION}

Local structural heterogeneities, due to Sb-doping and Gevacancy gathering have been identified as the seeds to reduce the domain size and to generate charged DWs. A localized point electrostatic field is created around the local structural heterogeneities, together with the positively and negatively charged 
DWs, forming a hierarchical internal electrostatic field that greatly enhances the power factor of the doped ferroelectric semiconductor GeTe. These findings make it possible for ferroelectric engineering to improve the thermoelectric performance in electronic and energy conversion devices.

\section{Received 16 November 2021; accepted 7 December 2021; published online 24 January 2022}

1 Chanthbouala A, Garcia V, Cherifi RO, et al. A ferroelectric memristor. Nat Mater, 2012, 11: 860-864

2 Chen Z, Li F, Huang Q, et al. Giant tuning of ferroelectricity in single crystals by thickness engineering. Sci Adv, 2020, 6: eabc7156

$3 \mathrm{Xu}$ R, Liu S, Grinberg I, et al. Ferroelectric polarization reversal via successive ferroelastic transitions. Nat Mater, 2015, 14: 79-86

4 Lee D, Jeon BC, Yoon A, et al. Flexoelectric control of defect formation in ferroelectric epitaxial thin films. Adv Mater, 2014, 26: 5005-5011

5 Martin LW, Rappe AM. Thin-film ferroelectric materials and their applications. Nat Rev Mater, 2017, 2: 16087

6 Scott JF. Soft-mode spectroscopy: Experimental studies of structural phase transitions. Rev Mod Phys, 1974, 46: 83-128

7 Fei Z, Zhao W, Palomaki TA, et al. Ferroelectric switching of a twodimensional metal. Nature, 2018, 560: 336-339

8 Benedek NA, Birol T. 'Ferroelectric' metals reexamined: Fundamental mechanisms and design considerations for new materials. J Mater Chem C, 2016, 4: 4000-4015

9 Shi Y, Guo Y, Wang X, et al. A ferroelectric-like structural transition in a metal. Nat Mater, 2013, 12: 1024-1027

10 Kim TH, Puggioni D, Yuan Y, et al. Polar metals by geometric design. Nature, 2016, 533: 68-72

11 Wang Z, Zhang T, Ding M, et al. Electric-field control of magnetism in a few-layered van der Waals ferromagnetic semiconductor. Nat Nanotech, 2018, 13: 554-559

12 Li L, Gao P, Nelson CT, et al. Atomic scale structure changes induced by charged domain walls in ferroelectric materials. Nano Lett, 2013, 13: 5218-5223

13 Zhang Y, Zhang Y, Guo Q, et al. Characterization of domain distributions by second harmonic generation in ferroelectrics. npj Comput Mater, 2018, 4: 39

14 Seidel J, Martin LW, He Q, et al. Conduction at domain walls in oxide multiferroics. Nat Mater, 2009, 8: 229-234

15 Picozzi S. Ferroelectric Rashba semiconductors as a novel class of multifunctional materials. Front Phys, 2014, 2: 10

16 Liao WQ, Zhang Y, Hu CL, et al. A lead-halide perovskite molecular ferroelectric semiconductor. Nat Commun, 2015, 6: 7338

17 Ogawa N, Sotome M, Kaneko Y, et al. Shift current in the ferroelectric semiconductor SbSi. Phys Rev B, 2017, 96: 241203

18 Chen Z, Chen Z, Kuo CY, et al. Complex strain evolution of polar and magnetic order in multiferroic $\mathrm{BiFeO}_{3}$ thin films. Nat Commun, 2018, 9: 3764

19 Kolobov AV, Kim DJ, Giussani A, et al. Ferroelectric switching in epitaxial GeTe films. APL Mater, 2014, 2: 066101

20 Li J, Chen Z, Zhang X, et al. Electronic origin of the high thermoelectric performance of GeTe among the p-type group IV monotellurides. NPG Asia Mater, 2017, 9: e353

21 Liu WD, Wang DZ, Liu Q, et al. High-performance GeTe-based thermoelectrics: From materials to devices. Adv Energy Mater, 2020, 10: 2000367

22 Li J, Zhang X, Chen Z, et al. Low-symmetry rhombohedral GeTe thermoelectrics. Joule, 2018, 2: 976-987

23 Tsai YF, Wei PC, Chang L, et al. Compositional fluctuations locked by athermal transformation yielding high thermoelectric performance in GeTe. Adv Mater, 2021, 33: 2005612

24 Sadia Y, Ohaion-Raz T, Ben-Yehuda O, et al. Criteria for extending the operation periods of thermoelectric converters based on IV-VI compounds. J Solid State Chem, 2016, 241: 79-85

25 Meroz O, Ben-Ayoun D, Beeri O, et al. Development of $\mathrm{Bi}_{2} \mathrm{Te}_{2.4} \mathrm{Se}_{0.6}$ alloy for thermoelectric power generation applications. J Alloys Compd,
2016, 679: 196-201

26 Ben-Ayoun D, Sadia Y, Gelbstein Y. High temperature thermoelectric properties evolution of $\mathrm{Pb}_{1-x} \mathrm{Sn}_{x}$ Te based alloys. J Alloys Compd, 2017, 722: $33-38$

27 Madar N, Givon T, Mogilyansky D, et al. High thermoelectric potential of $\mathrm{Bi}_{2} \mathrm{Te}_{3}$ alloyed GeTe-rich phases. J Appl Phys, 2016, 120: 035102

28 Sadia Y, Madar N, Kaler I, et al. Thermoelectric properties of the quasibinary $\mathrm{MnSi}_{1.73}-\mathrm{FeSi}_{2}$ system. J Elec Materi, 2015, 44: 1637-1643

29 Bauer Pereira P, Sergueev I, Gorsse S, et al. Lattice dynamics and structure of GeTe, SnTe and PbTe. Phys Status Solidi B, 2013, 250: 1300-1307

30 Vermeulen PA, Kumar A, ten Brink GH, et al. Unravelling the domain structures in GeTe and $\mathrm{LaAlO}_{3}$. Cryst Growth Des, 2016, 16: 5915-5922

31 Dangić $Đ$, Fahy S, Savić I. Giant thermoelectric power factor in charged ferroelectric domain walls of GeTe with Van Hove singularities. npj Comput Mater, 2020, 6: 195

$32 \mathrm{Wu} \mathrm{D}$, Xie L, Xu X, et al. High thermoelectric performance achieved in $\mathrm{GeTe}-\mathrm{Bi}_{2} \mathrm{Te}_{3}$ pseudo-binary via van der Waals gap-induced hierarchical ferroelectric domain structure. Adv Funct Mater, 2019, 29: 1806613

33 Meier D, Seidel J, Cano A, et al. Anisotropic conductance at improper ferroelectric domain walls. Nat Mater, 2012, 11: 284-288

34 Wang WY, Tang YL, Zhu YL, et al. Atomic level 1D structural modulations at the negatively charged domain walls in $\mathrm{BiFeO}_{3}$ films. Adv Mater Interfaces, 2015, 2: 1500024

35 Jin Y, Xiao S, Yang JC, et al. Conductive tail-to-tail domain walls in epitaxial $\mathrm{BiFeO}_{3}$ films. Appl Phys Lett, 2018, 113: 082904

36 Vasudevan RK, Cao Y, Laanait N, et al. Field enhancement of electronic conductance at ferroelectric domain walls. Nat Commun, 2017, 8: 1318

37 Zhang $\mathrm{Y}, \mathrm{Lu} \mathrm{H}$, Yan $\mathrm{X}$, et al. Intrinsic conductance of domain walls in $\mathrm{BiFeO}_{3}$. Adv Mater, 2019, 31: 1902099

38 Zhang X, Li J, Wang X, et al. Vacancy manipulation for thermoelectric enhancements in GeTe alloys. J Am Chem Soc, 2018, 140: 15883-15888

39 Li F, Cabral MJ, Xu B, et al. Giant piezoelectricity of Sm-doped $\mathrm{Pb}\left(\mathrm{Mg}_{1 / 3} \mathrm{Nb}_{2 / 3}\right) \mathrm{O}_{3}-\mathrm{PbTiO}_{3}$ single crystals. Science, 2019, 364: 264-268

40 Bayikadi KS, Wu CT, Chen LC, et al. Synergistic optimization of thermoelectric performance of $\mathrm{Sb}$ doped $\mathrm{GeTe}$ with a strained domain and domain boundaries. J Mater Chem A, 2020, 8: 5332-5341

Acknowledgements The work was supported by the National Natural Science Foundation of China (52072282), the National Key Research and Development Program of China (2019YFA0704900). The S/TEM work was performed at the Nanostructure Research Center (NRC), which is supported by the Fundamental Research Funds for the Central Universities (WUT: 2021III016GX).

Author contributions Meng X collected and analyzed the S/TEM and insitu S/TEM data. Chen S synthesized the materials. Bai H collected the S/ TEM data. Peng H performed the DFT calculations. Zhang $S$ designed the experiments and analyzed the ferroelectric data. Su X designed and performed the materials synthesis. Tan $G$ performed the materials synthesis. Sun $\mathrm{Z}$ performed the materials synthesis. Tendeloo GV analyzed the TEM data. Tang X designed the project and analyzed the data. Zhang Q conceived the project and analyzed the data. Wu J conceived the project, designed the experiments, analyzed the data and wrote the manuscript. All of the authors discussed and revised the manuscript before submission.

Conflict of interest The authors declare that they have no conflict of interest.

Supplementary information Supporting data are available in the online version of the paper. 


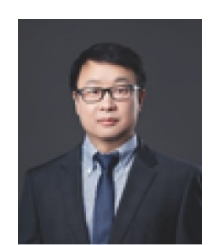

Jinsong Wu earned his $\mathrm{PhD}$ degree from the Department of Materials Science and Engineering at Dalian University of Technology. He is currently a professor and executive director of the Nanostructure Research Center at Wuhan University of Technology. Wu's research interests include transmission electron microscopy, electron tomography, in-situ transmission electron microscopy and nanomaterials for energy storage.

Xiangyu Meng received his BS degree in 2018 from Qilu University of Technology. He is currently pursuing his Master degree at Wuhan University of technology under the supervisor of Prof. Wu and Prof. Zhang. His research focuses on ferroelectric and thermoelectric materials.

\section{铁电工程: 铁电局部结构异质提高热电性能}

孟祥雨 ${ }^{1,2}$, 陈硕 ${ }^{1}$, 彭吴阳 ${ }^{1,2}$, 白辉 ${ }^{1,2}$, 张树君 ${ }^{3}$, 苏贤礼 ${ }^{1}$, 谭刚健 ${ }^{1}$, Gustaaf Van Tendeloo ${ }^{2,4}$, 孙志刚 ${ }^{1}$, 张清杰 ${ }^{1}$, 唐新峰 ${ }^{1}$, 吴劲松 $12^{*}$

摘要 $\mathrm{GeTe}$ 是一种典型的铁电半导体, 其性能可以通过掺杂各种元素 进行优化. 然而, 由于难以直接观察铁电畴及其在温度和电流的实际工 作条件下的演变, 因此铁电畴壁对热电性能的影响仍然未知. 本工作基 于对未掺杂的和 $S b$ 掺杂的 $\mathrm{GeTe}$ 晶体中铁电畴和铁电畴壁的原位实验 研究, 探究了铁电畴和铁电畴壁在外加电场和温度下的动态演化. 由于 掺杂引入的 $\mathrm{Sb}^{3+}$ 和 $\mathrm{Ge}$ 空位的相互作用, 产生了局部结构异质性, 引入纳 米尺寸的铁电畴, 使畴尺寸减小、带电畴壁密度增大, 从而大大提高了 热电性能. 这项工作揭示了铁电畴壁增强热电半导体性能的基本机制, 为高性能热电材料的发展提供了新自由度. 